Trabajos Originales

\title{
REPARACIÓN DE PROLAPSO VAGINAL ANTERIOR CON MAШA DE PROLENE
}

\author{
Humberto Hott A. 1,2, Roberto Iglesias R. ${ }^{1,2}$, Emil Schneider B. ${ }^{1,2}$, Denisse Anrique Ch. ${ }^{1}$, \\ Carola Villanueva M. ${ }^{a}$, Gonzalo Valdevenito A. ${ }^{b}$ \\ ${ }^{1}$ Instituto Obstetricia y Ginecología, Universidad Austral de Chile. ${ }^{2}$ Servicio Obstetricia y Ginecología, Hospital Base de \\ Valdivia.
}

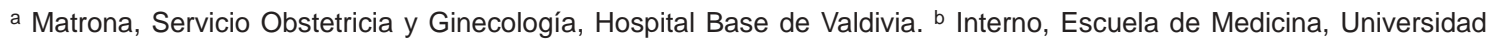
Austral de Chile.

\section{RESUMEN}

Antecedentes: La reparación del prolapso vaginal anterior presenta una alta tasa de recurrencia. La colporrafia anterior con malla de prolene es una buena alternativa quirúrgica que ha obtenido buenos resultados a largo plazo. Objetivo: Evaluar el resultado anatómico y la evolución de la colporrafia anterior con malla de prolene. Método: Estudio de cohorte prospectivo para evaluar el resultado quirúrgico. Resultados: Ingresaron 35 pacientes con una edad promedio de 61,4 años; $85,7 \%$ de ellas estaba en postmenopausia y $3 \%$ utilizaba terapia hormonal de reemplazo. La principal patología asociada fue la incontinencia de orina de esfuerzo (54,3\%). El estado del piso pelviano preoperatorio correspondió a 31,4\% cistocele grado II, $48,6 \%$ cistocele grado III y $20 \%$ cistocele grado IV. Se asoció prolapso uterino en $88,6 \%$ de los casos y $74,3 \%$ con prolapso de la pared posterior. No hubo complicaciones intraoperatorias. Hubo complicaciones postoperatorias en 4 pacientes $(11,4 \%)$. Una paciente presentó erosión de la malla $(2,9 \%)$. El seguimiento fue de 1 a 11 meses. En la evaluación postoperatoria de las pacientes con cistocele grado II $80 \%$ corrigió a grado 0; para cistocele grado III $72 \%$ corrigió a grado 0 . Un $11 \%$ llegó a grado I y $17 \%$ grado II. Para el grupo con cistocele grado IV, $71 \%$ resultó en grado 0 y $29 \%$ grado II. Conclusión: La técnica presentada solucionó un severo problema de calidad de vida de las pacientes afectadas. Se requiere un mayor número de casos y tiempo de seguimiento para su recomendación definitiva.

\section{PALABRAS CLAVE: Prolapso vaginal anterior, malla de prolene, cistocele}

\section{SUMMARY}

Background: Repair of anterior vaginal prolapse present a high rate of recurrence. Anterior colporrhaphy plus prolene mesh is a surgical option with optimal long term results. Objective: To evaluate anatomical results and evolution of anterior colporrhaphy with prolene mesh. Method: A prospective cohort study was made to evaluate the surgical outcomes. Results: 35 patients were recruited with a median age of 61.4 years. $85.7 \%$ of them were in postmenopausal status and $3 \%$ were using hormone replacement therapy. Stress urinary incontinence was associated in $54.3 \%$ of the cases. The pelvic floor stage at entry was $31.4 \%$ cysthocele stage II, $48.6 \%$ stage III and $20 \%$ cysthocele stage IV. Uterine prolapse was present in $88.6 \%$ of cases and the posterior wall was compromised in $74.3 \%$. There were no intraoperatory complications. Postoperatory complications occurred in 4 patients $(11.4 \%)$. Only in one case the mesh eroded $(2.9 \%)$. The follow up was from 1 to 11 months. Of the patients with cysthocele stage II at the moment of evaluation, $80 \%$ were at stage 0 . For cysthocele stage III $72 \%$ turned to stage $0.11 \%$ turned to stage I and $17 \%$ to stage II. The cases that 
presented cysthocele stage IV $71 \%$ were at stage 0 and $29 \%$ at stage II. Conclusion: This technique solved an important quality of life problem of the affected patients. A higher number of cases and time of follow up is needed to recommend this technique definitely.

\section{KEY WORDS: Anterior vaginal prolapse, prolene mesh, cysthocele}

\section{INTRODUCCIÓN}

El riesgo a lo largo de la vida de que una mujer sea operada por prolapso es de $11 \%$ y aproximadamente un tercio requiere reintervención quirúrgica (1). Se ha estimado que la demanda de esta cirugía aumentaría alrededor de $45 \%$ en los próximos 30 años, dado por cambios demográficos (2).

La reparación del prolapso vaginal anterior representa uno de los aspectos más desafiantes de la cirugía reconstructiva pelviana en términos de alcanzar un adecuado resultado y que este se mantenga a largo plazo. Se ha descrito recurrencia posterior a colporrafia anterior de hasta $40 \%(3,4)$. Esto ha llevado al perfeccionamiento de las técnicas y a la utilización de mallas sintéticas. Su función se basa principalmente en reforzar o reemplazar zonas de tejido dañado $(5,6)$. Polipropileno constituye el mejor material disponible, ya que es biocompatible, inerte y resistente. Un estudio de 12 pacientes intervenidas mediante reparación de cistocele con malla de prolene obtuvo como resultado un $100 \%$ de curación (7). El único estudio randomizado que compara diferentes técnicas quirúrgicas obtuvo resultados óptimos en $42 \%$ intervenciones con malla, comparado con un $30 \%$ en el grupo colporrafia anterior estándar (8). Estudios recientes han obtenido tasas más bajas de recurrencia (6\%) (9). La principal complicación corresponde a erosión de la malla, presente entre 9 y $25 \%$ de los casos (10).

El objetivo de este estudio es evaluar el resultado anatómico y la evolución de la colporrafia anterior con malla de prolene.

\section{PACIENTES Y MÉTODO}

Se realizó un estudio de cohorte prospectivo de pacientes con prolapso vaginal anterior, intervenidas mediante colporrafia anterior (CA) con malla de prolene en la Unidad de Ginecología del Hospital Base Valdivia. El protocolo se inició el 19 julio de 2005 y se incluyeron pacientes hasta el 29 de mayo 2006. Los criterios de inclusión fueron: 1. cistocele grado II o mayor, 2. primario o recidivado, y 3. sin contraindicación quirúrgica.

El estado del piso pelviano fue evaluado mediante examen físico utilizando la clasificación POPQ
(Pelvic Organ Prolapse Quantification System) de la Sociedad Internacional de Continencia (11). Se analizaron las características de las pacientes (edad, origen, paridad, estado hormonal gonadal, uso de terapia de reemplazo hormonal, patología asociada). Todas las pacientes fueron intervenidas mediante la misma técnica por el equipo de cirugía de piso pelviano. Se administró profilaxis antibiótica preoperatorio (metronidazol $1 \mathrm{~g}$ vía rectal y cefazolina $1 \mathrm{~g} \mathrm{ev}$ ) al momento de la administración de la anestesia.

Técnica quirúrgica: Bajo anestesia regional, con la paciente en posición ginecológica y extremidades inferiores en hiperflexión se procede a realizar histerectomía vaginal en los casos que corresponde. Posteriormente, se expone la pared vaginal anterior y se infiltra con una solución de lidocaína $0,4 \%$ y epinefrina al $0,0002 \%$ para separar los planos de disección y mejorar la hemostasia. Se realiza una colpotomía anterior resecando un trozo de mucosa con vértice a nivel del cuello vesical. El ancho de la base varía según la cantidad de mucosa vaginal a resecar. El cistocele se diseca lateralmente en forma completa hasta ubicar la unión de la fascia con el arco tendíneo. Se perfora en forma digital o con tijera para acceder al espacio de Retzius. Se procede a reducir el cistocele mediante puntos de Vicryl 2.0 formando una gareta. Se instala una prótesis de polipropileno de $2 \mathrm{~cm}$ de ancho y la longitud necesaria para introducir ambos extremos en los túneles previamente tallados. Se deja sin fijación. Una vez que la vejiga es sostenida por la prótesis, la colporrafia se realiza con sutura de Vicryl 2.0. Esta cirugía se basa en la técnica descrita por De Tayrac (12). La intervención se completa en función del estado clínico de la paciente, realizando cirugía para incontinencia Trans Obturator Tape (TOT) y/o reparación del resto del piso pelviano. Se reevalúa el estado del piso pelviano en el postoperatorio y se cita a las pacientes a control al mes 3,6 y 11 .

\section{RESULTADOS}

Ingresaron 35 pacientes al estudio con una edad promedio de 61,4 años (rango: 39 a 83 años). 


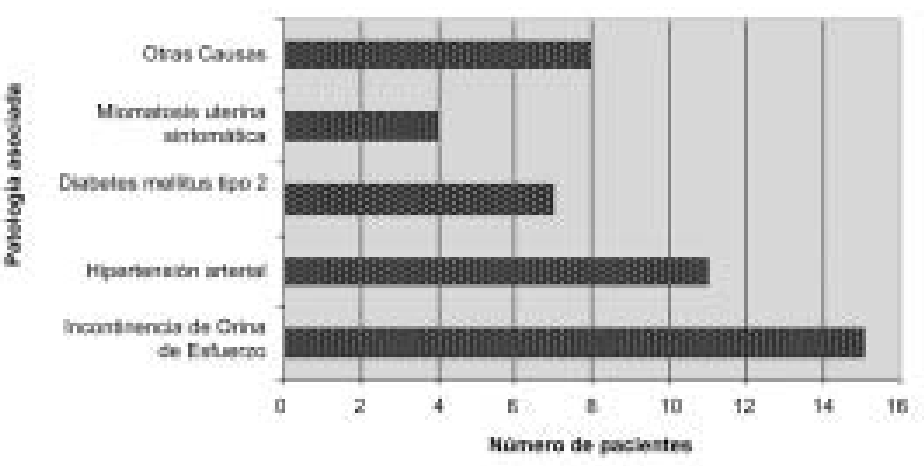

Figura 1. Número de casos según patología asociada, en pacientes intervenidas mediante colporrafia anterior con malla de prolene.
El origen de las pacientes fue $63 \%$ rural y $47 \%$ urbano. La paridad promedio del grupo fue 4,3 (rango: 0 y 13 hijos). Dos pacientes eran nulíparas $(5,7 \%)$. El estado hormonal gonadal al momento de la cirugía correspondió a postmenopausia en el $85,7 \%$ de las pacientes. La principal patología asociada fue la incontinencia de orina de esfuerzo $(54,3 \%)$, seguida de la hipertensión arterial $(31,4 \%)$ y la diabetes mellitus tipo $2(20 \%)$. Hubo 4 casos de miomatosis uterina sintomática (11,4\%) (Figura 1).

En cuanto al estado del piso pelviano preoperatorio $31,4 \%$ de las pacientes presentó cistocele grado II, $48,6 \%$ cistocele grado III y $20 \%$ cistocele grado IV. Además se asoció prolapso uterino en el $88,6 \%$ de los casos, de los cuales el $55 \%$ correspondía a grado III o IV. El prolapso de la pared posterior, se presentó en $74,3 \%$ de los casos; $23 \%$ correspondía a grado III o IV (Tabla I).

Se realizó histerectomía vaginal a 26 pacientes (74,3\%). Se reparó la pared posterior a 17 pacientes $(48,6 \%)$. La incontinencia de orina de esfuerzo se trató con TOT a 19 pacientes $(54,3 \%)$.

No hubo complicaciones intraoperatorias. Complicaciones postoperatorias se presentaron en 4 pacientes $(11,4 \%)$. Estas correspondieron a infección del tracto urinario bajo, retención urinaria y un

Tabla I

NÚMERO DE PACIENTES SEGÚN ESTRUCTURA ANATÓMICA COMPROMETIDA EN EL PREOPERATORIO DE ACUERDO A LA CLASIFICACIÓN DE LA SOCIEDAD INTERNACIONAL DE CONTINENCIA

\begin{tabular}{cccc}
\hline Grado & Pared anterior & Pared posterior & Útero \\
\hline 0 & 0 & 9 & 4 \\
I & 0 & 10 & 5 \\
II & 11 & 10 & 9 \\
III & 17 & 3 & 10 \\
IV & 7 & 3 & 7 \\
\hline
\end{tabular}

hematoma de la cúpula vaginal. Las pacientes fueron dadas de alta a los 2,7 días promedio (rango: 2 a 7 días). Sólo una paciente presentó erosión de la malla $(2,9 \%)$ y requirió recorte y sutura de vagina 2 meses después.

El seguimiento fue de 1 a 11 meses. En la evaluación postoperatoria de las pacientes con cistocele grado II hubo una corrección a grado 0 de un $80 \%$, y $20 \%$ se mantuvo grado II. Para cistocele grado III, $72 \%$ se corrigió a grado $0 ; 11 \%$ llegó a grado I y $17 \%$ a grado II. Para el grupo con cistocele grado IV, $71 \%$ resultó en grado 0 y $29 \%$ grado II (Tabla II y III).

En los casos en que se realizó TOT un 95\% de las pacientes se encontraba continente al momento del control.

\section{DISCUSIÓN}

Actualmente el prolapso vaginal constituye un problema de salud pública. Esto confiere gran relevancia al tratamiento quirúrgico y a las medidas enfocadas al perfeccionamiento de las técnicas para mejorar el resultado a largo plazo.

El grupo de pacientes evaluadas en este estudio presentó características demográficas simila-

Tabla II

NÚMERO DE PACIENTES SEGÚN ESTADO PRE Y POSTOPERATORIO DE LA PARED VAGINAL ANTERIOR DE ACUERDO A LA CLASIFICACIÓN DE LA SOCIEDAD INTERNACIONAL DE CONTINENCIA

\begin{tabular}{ccc}
\hline Grado & Preoperatorio & Postoperatorio \\
\hline 0 & 0 & 26 \\
I & 0 & 2 \\
II & 11 & 7 \\
III & 17 & 0 \\
IV & 7 & 0 \\
\hline
\end{tabular}


Tabla III

\section{ESTADO DEL PISO PELVIANO PRE Y POSTOPERA-} TORIO SEGÚN MESES DE EVALUACIÓN

\begin{tabular}{|c|c|c|}
\hline \multirow[b]{2}{*}{ Meses seguimiento } & \multicolumn{2}{|c|}{ Cistocele } \\
\hline & Preoperatorio & Postoperatorio \\
\hline 1 & III & 0 \\
\hline 1 & IV & 0 \\
\hline 2 & III & 1 \\
\hline 2 & III & 0 \\
\hline 2 & III & 0 \\
\hline 2 & III & 0 \\
\hline 2 & II & 0 \\
\hline 2 & II & 0 \\
\hline 3 & ॥ & 0 \\
\hline 3 & III & 0 \\
\hline 3 & III & 0 \\
\hline 3 & IV & 0 \\
\hline 4 & II & 0 \\
\hline 4 & IV & 0 \\
\hline 4 & IV & 0 \\
\hline 5 & II & 0 \\
\hline 5 & ॥ & 0 \\
\hline 5 & III & 0 \\
\hline 5 & IV & ॥ \\
\hline 5,5 & III & 0 \\
\hline 5,5 & III & 0 \\
\hline 5,5 & III & 1 \\
\hline 6 & II & 0 \\
\hline 6 & III & 0 \\
\hline 7 & ॥ & ॥ \\
\hline 7 & III & 0 \\
\hline 7,5 & II & II \\
\hline 7,5 & IV & II \\
\hline 8 & II & 0 \\
\hline 8 & III & 0 \\
\hline 8 & III & II \\
\hline 8 & III & II \\
\hline 8 & III & II \\
\hline 9 & II & 0 \\
\hline 11 & IV & 0 \\
\hline
\end{tabular}

res, comparado con trabajos publicados previamente $(8,9,14)$. Difiere en cuanto a que la mayoría se encontraba en la postmenopausia pero sin terapia hormonal de reemplazo (8). En cuanto a la paridad promedio, el valor es elevado, considerando que este en un factor de riesgo importante para prolapso genital $(15,16)$.

Se obtuvieron resultados óptimos en un alto porcentaje. Sin embargo, hay que reconocer que la principal limitación de este estudio está dada por el reducido número de pacientes incluidas y el poco tiempo de seguimiento. Pese a que en este estudio la tasa de erosión de la malla fue muy baja, esta patología sigue representando la principal complicación de la técnica (10). La reparación del prolapso vaginal anterior es un desafío para los ginecólogos, debido principalmente a la alta tasa de recurrencia. Para evaluar esto de forma adecuada se requiere un seguimiento prolongado, problema principal de los trabajos existentes $(7,8,9)$.

Existen múltiples técnicas para reparar el compartimento anterior y el desafío es mayor en la medida que aumentan las áreas comprometidas. Cuando el prolapso es severo o recurrente la reparación tradicional utilizando solo material de sutura es insuficiente en la mayoría de los casos. Es aquí cuando cobran importancia los materiales de soporte adicional. Las alternativas son: tejido autólogo, injertos de dermis de porcino, malla sintética absorbible y malla sintética permanente.

Actualmente no quedan dudas respecto al beneficio de la utilización de materiales protésicos sintéticos en la cirugía de prolapso. Al momento de elegir una malla sintética hay que considerar sus características. Idealmente debe ser flexible para que disminuya la tensión al instalarla y se amolde fácilmente. Liviana y resistente para que otorgue el soporte necesario. El tamaño del poro debe ser mayor de 75 micrones para que se produzcan vasos de neoformación y se acoplen distintas células a la estructura de la malla. Además debiera ser biocompatible. El material ideal aún no existe, sin embargo el polipropileno parece ser la mejor alternativa disponible en la actualidad.

Hay que considerar que la posición en la que se instala la malla influye en el resultado tanto anatómico como funcional. El uso de extensiones hacia la pared de la pelvis otorga un mejor soporte.

Trabajos que estudian la efectividad del tratamiento han considerado como resultado óptimo punto Aa estadio $0(-3 \mathrm{~cm})$ y satisfactorio en estadio I $(-2 \mathrm{~cm})$ (8). La mayoría de los casos en que se realizó CA la pared vaginal anterior quedó a nivel del himen (grado II); si bien esto pudiera considerarse fracaso terapéutico según la definición antes mencionada, la tasa de síntomas en estas pacientes es baja y por lo tanto están conformes con los resultados y su calidad de vida actual. En esta experiencia el $20 \%$ de las pacientes resultaron con cistocele grado II y todas se encontraban satisfechas con los resultados.

\section{CONCLUSIÓN}

La técnica presentada solucionó un severo problema de calidad de vida de las pacientes afectadas. Es simple y de bajo costo. Se requiere un 
mayor número de casos y tiempo de seguimiento para su recomendación definitiva. Su realización corresponde a ginecólogos con entrenamiento en ginecología urológica.

\section{BIBLIOGRAFÍA}

1. Olsen AL, Smith VJ, Bergstrom JO, Colling JC, Clarck AL. Epidemiology of surgically managed pelvic organ prolapse and urinary incontinence. Am J Obstet Gynecol 1997;89(4):501-6.

2. Luber KM, Boero S, Chloe JY. The demographics of pelvic floor disorders: current observations and future projections. Am J Obstet Gynecol 2001;184:1496501.

3. Graves EJ, Gillum BS. National hospital discharge survey: annual summary, 1994. National Center for Health Statistics. Vital Health Stat 13 1997;(128): i-v; 1-50.

4. Beck P, McCormick S. Treatment of urinary stress incontinence with anterior colporrhaphy. Obstet Gynecol 1982;59:269-74.

5. Scales JT. Materials for hernia repair. Proc R Soc Med 1953;46:647-52.

6. Iglesia CB, Fenner DE, Brubaker L. The use of prosthetic materials in the repair of hernias. Surg Clin North Am 1971;51:1287-99.

7. Migliari R, De Angelis M, Madeddu G, Verdacchi T. Tension free vaginal mesh repair for anterior vaginal prolapse. Eur Urol 2000;38:151-5.

8. Weber AM, Walters MD. Anterior colporrhaphy: a randomized trial of three surgical techniques. Am J Obstet Gynecol 2001;185:1299-304.

9. Dwyer PI, O'Reilly BA. Transvaginal repair of anterior and posterior compartiment prolapse with atrium polypropylene mesh. BJOG 2004;111(8):831-6.

10. Cuneo B, Veau V. De la signification morphologique des aponeuroses perivescales. J Anat Phisiol 1982; 59:269-74.

11. Bland DR, Earle BB, Vitolins MZ, Burke G. Use of the pelvic organ prolapse staging system of the International Continence Society, American Uroginecologic Society, and Society of Gynecologic Surgeons in perimenopausal woman. Am J Obstet Gynecol 1999; 181(6):1324-7.

12. De Tayrac R., Gervaise A, Fernandez H. Cystocele repair by the vaginal route with a tension free sub bladder prothesis. J Gynecol Obstet Biol Reprod 2002; 31(6):597-9.

13. Milani R, Salvatore $S$, Soligo M, Pifarotti $P$, Meschia $\mathrm{M}$, Cortese M. Functional and anatomical outcome of anterior and posterior vaginal prolapse repair with prolene mesh. Br J Obstet Gynaecol 2005;112(1):10711.

14. Smith P, Heimer G, Norgen A, et al. Steroid Hormone Receptors in Pelvic Muscles and Ligament in Women. Gynecol Obstet Invest 1990;30:27-30.

15. Gilpin SA, Gosling JA, Smith ARB, et al. The pathogenesis of genitourinary prolapse and stress incontinence of urine. A histological and histochemical study. Br J Obstet Gynecol 1989;96:15-23.

16. Norton PA, Baker JE, Sharp HC, et al. Genitourinary prolapse and joint hypermobility in women. Obstet Gynecol 1995;85(2):225-8. 